\title{
CUESTIÓN SOCIAL Y RELACIONES FAMILIARES: APROXIMACIÓN A LAS TENDENCIAS EN LAS políticas SOCIALES en América Latina
}

\author{
SOCIAL AFFAIR AND FAMILY RELATIONSHIPS: AN APPROACH TO \\ THE TRENDS OF SOCIAL POLICY IN LATIN AMERICA
}

\author{
Por: Manuel W. Mallardi* \\ María Paola Musso** \\ Marian González** \\ Recibido 25 octubre 2013. Aprobado 26 febrero 20014
}

* MANUEL MALLARDI Doctor en Ciencias Sociatura en Trabajo Social de la Universidad Nacional del Centro de la Provincia de Buenas Aires.

**MARIAN GONZÁLEZ Y MARIA PAOLA MUSSO Estudiantes licenciatura Estudiantes licenciatura sida Nacial. Univerde la Provincia

\section{RESUMEN}

El presente artículo socializa resultados de investigación sobre las tendencias en las políticas sociales en América Latina, haciendo especial referencia a las consideraciones que éstas tienen en torno a la familia y las relaciones sociales que en su interior se desarrollan. Para ello, el trabajo sintetiza el análisis de los principales mecanismos que adquiere la intervención contemporánea sobre la "cuestión social" en los Programas de Transferencia de Renta Condicionada: la corresponsabilidad, la familiarización y la maternalización de la política social.

El análisis se centra en la revisión de producciones y documentos emanados de organismos internacionales cuyas posturas presentan relevancia e influencia en materia de política social en América Latina: la Comisión Económica para América Latina y el Caribe (CEPAL) y el Banco Mundial. Ambos organismos presentan una fructífera producción sobre los Programas de Transferencia de Renta Condicionada, como así también mecanismos de incidencia en las agendas nacionales, lo cual otorga relevancia al análisis de los mencionados documentos.

Palabras Clave: Cuestión social - programas de transferencia de renta condicionada - América Latina - organismos internacionales

\begin{abstract}
This article socialize results in research of Latin America social politic trends, having in special reference to the consideration this one has around family and social relationships that are developed below. For that, the paper summarizes the analysis of the main mechanisms that acquires the "contemporary" intervention on the "social question" in the Conditional Transfer Rent Programs: the co-responsibility, familiarization and maternalization of the social politic.
\end{abstract}

The analysis focuses on the revisions of produccions and documents sent by international organisms whose positions present relevance and influence in Latin America's social politics mathers: Economic Comission for Latin American and the Caribbean (ECLAC) and the World Bank. Both organisms present a succesfull production over Conditional Transfer Rent Program, as well as mechanisms of influence at national agendas, which gives relevance analysis to the mentioned documents.

Keywords: Social question - Conditional Transfer Rent Program - Latin America - International Organisms 


\section{Introducción}

$\sqrt{ }$

n términos socio-históricos la intervención de los estados capitalistas sobre las distintas expresiones de la "cuestión social" se ha caracterizado por la articulación de estrategias de coerción y de consenso, donde la primacía de una u otra forma de intervención se constituye en el resultado de un complejo interjuego de luchas y tensiones entre los sectores sociales fundamentales de la sociedad.

En este marco, las políticas sociales adquieren relevancia en los Estados capitalistas democráticos como formas sistemáticas de intervención y construcción de hegemonía por los sectores dirigentes. De este modo, desde las primeras experiencias hasta la actualidad, la política social se asocia a la configuración socio-histórica de la ciudadanía, principalmente en lo que a la consideración de derechos sociales respecta.

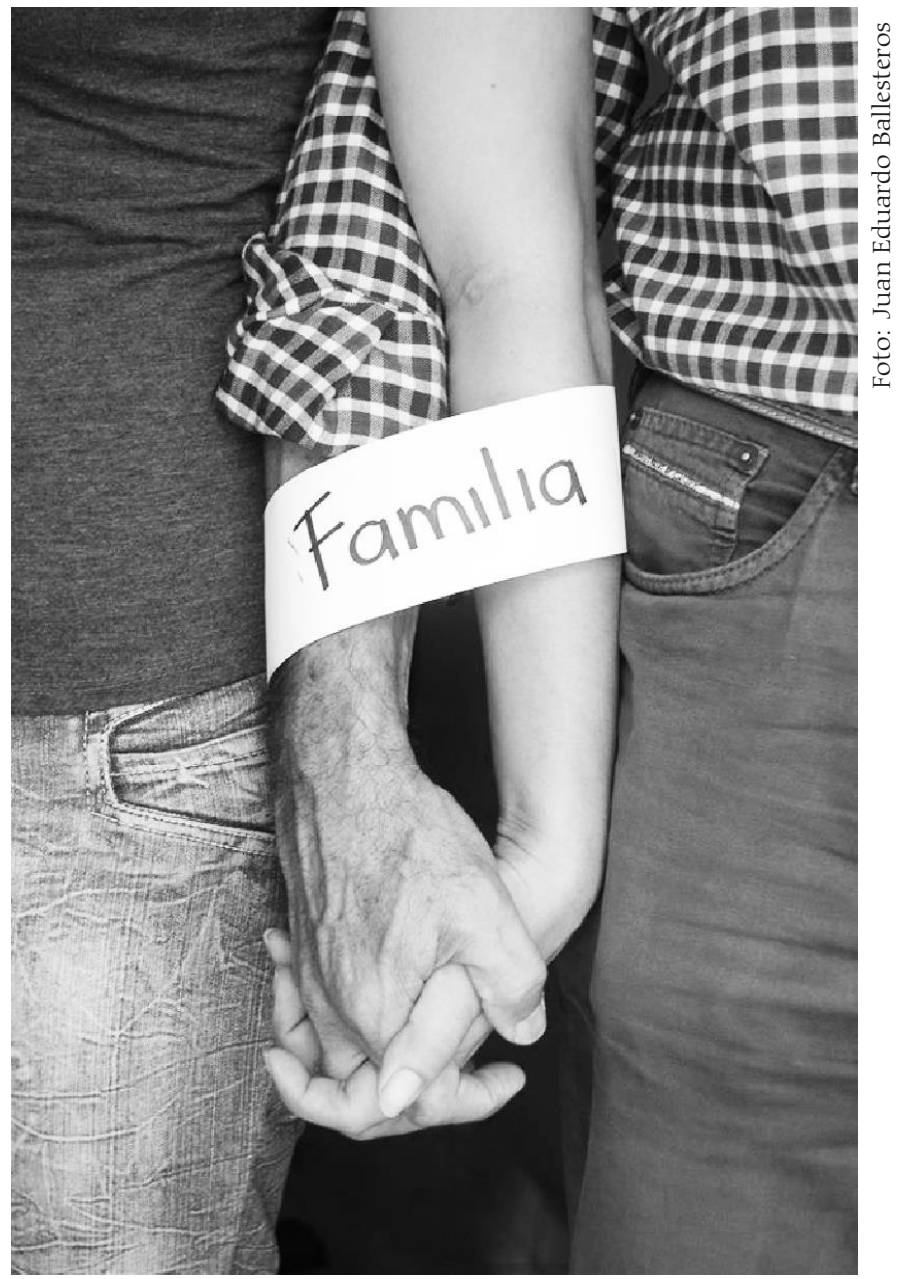

Teniendo en cuenta estos elementos y profundizando el estudio de las estrategias contemporáneas de intervención sobre la "cuestión social", el artículo propone profundizar la indagación sobre las políticas asistenciales implementadas por los Estados nacionales de América Latina, haciendo especial referencia a los Programas de Transferencia de Renta Condicionada. Para ello, se realiza una revisión de las producciones bibliográficas y documentales desarrolladas por organismos internacionales cuyas posturas tienen ingerencia en la agenda de las políticas sociales en la región: la Comisión Económica para América Latina y el Caribe (CEPAL) y el Banco Mundial.

\section{Cuestión social y políticas sociales: principales elementos analíticos}

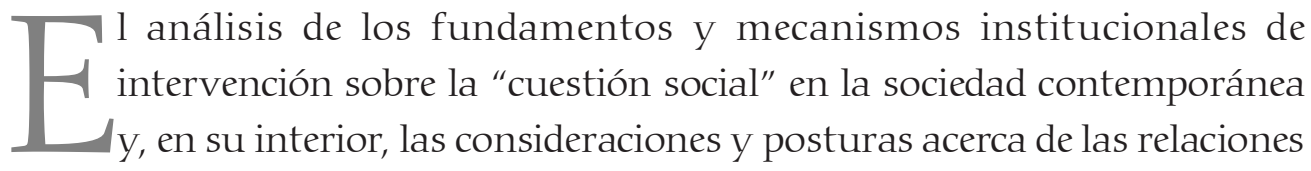
familiares, exige mencionar brevemente algunos elementos analíticos acerca 
de las implicaciones socio-históricas de la "cuestión social".

En tanto categoría polisémica, pues coexisten distintas posturas acerca de su significado e implicancias, la necesidad de precisar qué se entiende por "cuestión social" está dada porque la reconstrucción analítica que se desarrolle tendrá repercusiones en las formas de entender las políticas públicas en general y las políticas sociales en particular.

Al interior de las explicaciones sobre los fundamentos e influencias de la "cuestión social", la perspectiva analítica adoptada recupera la relación intrínseca entre la génesis de la "cuestión social" y las relaciones sociales capitalistas, reconociendo valiosos aportes en las producciones de referentes del Trabajo Social y las Ciencias Sociales en América Latina (Netto, 2002; Iamamoto, 1997; y Oliva, 2007). Estas producciones centran la discusión en los fundamentos y expresiones contemporáneas del proceso de pauperización propio de las relaciones sociales capitalistas, concluyendo que la "cuestión social" representa una expresión de la contradicción entre capital y trabajo. Esta postura se basa en el análisis del origen relacional del proceso de empobrecimiento de amplios sectores de la población en la sociedad capitalista, en tanto que, en los modos de producción anteriores la pobreza se asociaba principalmente a la escasez, mientras que en la sociabilidad burguesa el enriquecimiento de unos supone el empobrecimiento de otros.

Teniendo en cuenta estas reflexiones, en otro trabajo se sintetizaron las características fundamentales de la "cuestión social" para esta aproximación analítica: a) es producto de la instauración del modo de producción capitalista; b) supone una tendencia total que afecta de manera particular y diferenciada a distintos sectores de la población; c) implica el empobrecimiento de la clase trabajadora en relación con el enriquecimiento de los sectores capitalistas; y d) es consecuencia de la movilización y reivindicaciones de distintos sectores y fracciones que suponen el pasaje de una clase trabajadora con conciencia en-si a para-si, es decir, la conformación de un actor político fundamental en la sociedad burguesa (Mallardi, 2012).

El surgimiento y la consolidación de la "cuestión social" exige un nuevo rol histórico de los Estados modernos, imbricando en su accionar funciones económicas y políticas, entre las que destacamos la gestión de la fuerza de trabajo (Netto, 2002). Siguiendo a Torrado (2003), la gestión de la reproducción de la fuerza de trabajo supone un proceso interventivo que desarrolla el Estado 
en las sociedades capitalistas para crear, organizar y administrar instituciones que aseguren la producción de "trabajadores libres" y que tomen a su cargo los costos de reproducción de la fuerza de trabajo que no son considerados por el capital. Se trata, así, de un conjunto de intervenciones sociales del Estado que regulan indirectamente la forma mercancía de la fuerza de trabajo mediante distintos mecanismos de distribución secundaria del ingreso (Danani, 2009).

Entonces, en el marco de la profundización de los procesos de pauperización, la preservación y control de la fuerza de trabajo, ocupada y excedente, se constituye en una función estatal de primer orden. Por ello, en un contradictorio proceso de legitimación de los sectores dominantes y ampliación de la ciudadanía de los sectores trabajadores, las secuelas de la "cuestión social" pasan a constituirse en objeto de políticas sociales estatales.

Estas tensiones asocian las luchas y reivindicaciones sociales con la instauración de un sistema democrático, y sus consecuentes procesos de ciudadanía. Desde esta perspectiva, esta se constituye en el resultado momentáneo de los procesos sociales conflictivos que caracterizan a la sociedad capitalista, siendo la capacidad conquistada de apropiarse de los bienes socialmente creados. Así, los aspectos civiles, políticos y sociales que componen la ciudadanía moderna deben ser pensados tanto como concesiones del Estado democrático como conquistas de las clases trabajadoras (Coutinho, 1997). Ciertamente, el autor sostiene que "los derechos tienen siempre su primera expresión sobre la forma de expectativas de derecho, o sea, de demandas que son formuladas, en un momento histórico determinado, por clases o grupos sociales" (1997: 148). De este modo, la ciudadanía implica una conjunción de derechos obtenidos a partir de la correlación de fuerzas existentes, los cuales componen el llamado Estado de derecho democrático, el cual involucra, por un lado, el voto popular como forma de acceder al poder, mientras que por el otro exige el control social de la administración pública.

Al interior del proceso de gestión de la reproducción de la fuerza de trabajo, que articula políticas sanitarias, educativas, demográficas, sociales, entre otras, interesa profundizar el estudio de las políticas asistenciales, en tanto son instancias que crean mecanismos de transferencia de bienes y servicios a sujetos, individuales y colectivos, que no son absorbidos por el mercado de trabajo o cuya absorción es precaria o irregular (Soldano y Andrenacci, 2006).

Recuperando la trayectoria histórica en América Latina, donde las políticas 
asistenciales se materializan predominantemente en Programas de Transferencia de Renta Condicionadas, se observa que la institucionalidad social encargada de diseñar y ejecutar este tipo de políticas es variada, cubriendo un abanico que incluye instancias ministeriales, secretarias hasta la ausencia de instituciones responsables (Arriagada, 2007). Sin embargo, la gestión de este tipo de políticas asistenciales se encuentra presente en la totalidad de los países, como son los casos de: Plan Bolsa Familia en Brasil, Asignación Universal por Hijo en Argentina, el Bono Juancito Pinto en Bolivia, Plan Oportunidades en México, Bono de Desarrollo Humano en Ecuador, Programa Juntos en Perú, entre otros. A partir del análisis de los fundamentos y lógica de los mencionados programas surge un conjunto de interrogantes que orientan la continuidad del trabajo y que se vinculan a las concepciones que estas políticas tienen y/o intentan construir de las relaciones familiares. Al respecto, en el análisis de las distintas expresiones de la "cuestión social" que realizan los diferentes programas, nos preguntamos acerca de cuáles les atribuyen a las familias en general y a cada uno de sus miembros en particular por las situaciones vividas que interpelan su cotidiano, y, en relación a este punto, surge el interrogante asociado a cómo se expresa el discurso de participación de la población usuaria en los distintos programas.

Procurando avanzar en las respuestas a estos interrogantes, y con el fin de realizar una reconstrucción de las tendencias que adquieren estos mecanismos en América Latina, el trabajo analiza las producciones realizadas por instituciones supranacionales que se constituyen en referencia para los distintos gobiernos de la región: la Comisión Económica para América Latina y el Caribe (CEPAL) y el Banco Mundial.

Con el fin de ordenar la exposición se presentan tres mecanismos que coexisten en las políticas asistenciales, donde, si bien en los distintos casos se puede visualizar la relevancia de uno u otro, su articulación es una particularidad de las estrategias actuales de intervención sobre la "cuestión social". Estos mecanismos son: la corresponsabilidad, la familiarización, y la maternalización.

\section{La corresponsabilidad como política de Estado}

En los últimos años se han realizado cambios sustanciales en la legislación y en la política pública de América Latina que involucra a diversos sectores de la población. La incorporación de discusiones internacionales en torno a determinadas problemáticas, la participación e incidencia de las distintas 
expresiones de la Sociedad Civil en la definición y ejecución de las políticas públicas han incorporado a las estrategias institucionales de intervención sobre la "cuestión social" el planteamiento de la corresponsabilidad, el cual se expresa en dos acepciones distintas pero complementarias. Por un lado, la corresponsabilidad aparece como un elemento legitimador de la participación de la Sociedad Civil en las políticas públicas y, por el otro, se constituye como un horizonte en la intervención con los ciudadanos, planteando distintos grados de responsabilidad en las causas de las situaciones problemáticas que interpelan su cotidianidad, y, por lo tanto, en las posibles soluciones.

Teniendo en cuenta estas dos acepciones, en este artículo el interés se centra en la segunda forma, es decir comprender la corresponsabilidad en sus distintos niveles, en tanto un discurso que, como veremos a continuación, trastoca los alcances de la noción de ciudadanía.

Comenzando el análisis de los documentos de los organismos mencionados, se observa, por ejemplo, que en el año 2006 la CEPAL planteaba la necesidad de introducir y fortalecer el peso de la corresponsabilidad a partir de una idea de ciudadanía que plantea derechos y obligaciones a los miembros de una comunidad. De este modo, las exigencias relacionadas al cumplimiento de condicionalidades que plantean determinadas políticas sociales, particularmente los Programas de Transferencia de Renta Condicionada, estarían vinculadas al par derecho/obligación que conformarían la ciudadanía moderna, en la cual se hace necesaria la promoción de actitudes responsables a largo plazo por parte de personas que acceden a los programas. En el mencionado documento se menciona que

las principales innovaciones radican en la primordial importancia otorgada a la corresponsabilidad de las familias beneficiarias; la condicionalidad concebida como incentivo llevado al plano familiar (no como mecanismo de autoselección y focalización) y como elemento de articulación de metas a corto y largo plazo; la prioridad explícita otorgada a la búsqueda de sinergias sectoriales con fines de acumulación de capital humano, no solo de los niños de edad escolar sino también en la primera infancia, y el papel preponderante que se ha dado a la mujer en la recepción de los beneficios y en la participación en la aplicación y seguimiento. ${ }^{1}$ (CEPAL, 2006: 165-166)

Podemos encontrar posturas similares en instituciones que tienen una permanente incidencia en las políticas sociales de América Latina, como es el
${ }^{1} \mathrm{El}$ documento agrega que "desde esta perspectiva, para que los niños y las niñas puedan ejercer plenamente el el derecho a la educación, los padres tienen que evitar la deserción escolar. Si en determinadas situaciones el costo de oportunidad lleva a familias muy pobres a retirar a los hijos de la escuela para ocuparlos en actividades que contribuyen al ingreso familiar, es necesario enfrentar este problema a fin de mejorar la capacidad de respuesta de los padres con relación al derecho a la educación de los hijos". (CEPAL, 2006, 172-173) 
${ }^{2}$ La noción que los programas de TMC constituyen una nueva forma de contrato social entre el Estado y los beneficiarios se ha manifestado en uso del término en el uso del término corresponsabilidades (en lugar mán de progras moría de programas, tina (Fiszbein Schar,

${ }^{3}$ Se supone que ese ejercicio de cumplir las contraprestaciones contribuirá a que las familias aumenten la seguridad en sí mismas, incrementen su responsabilidad sobre el futuro del grupo y de las nuevas generaciones, $y$ adquieran habilidades para acceder a instancias administrativas y de mercado que les permitan avanzar en la solución autónom sus problemas (Cohen y Franco, 2006: 44-45). caso del Banco Mundial. En un informe elaborado por Fiszbein y Schady se plantean una serie de argumentos que tienden a justificar las políticas sociales con corresponsabilidad, entre los cuales se menciona que los ciudadanos que aportan mediante distintos impuestos a la recaudación estatal estarían más interesados en apoyar el diseño y ejecución de políticas sociales destinadas a personas que se están ayudando a sí mismos, en lugar de a otras personas igualmente pobres que se consideran como perezosas o descuidadas. De este modo, la corresponsabilidad viene a plantear un tipo de asistencia social que establece un nuevo tipo de "contrato social mediante el cual la sociedad (a través del Estado) apoya a las familias pobres que están dispuestas a hacer el esfuerzo de "mejorar sus vidas", es decir, los pobres meritorios"2" (2009: 63)

Esta postura plantearía una nueva relación del Estado para con los ciudadanos, en tanto que cuando "las condiciones se consideran como corresponsabilidades, tratan al receptor más como un "adulto", capaz de resolver sus propios problemas. El Estado es un socio en el proceso y no una nodriza” (Ibíd.: 66).

Por su parte, la postura de dos intelectuales que apoyan la implementación de estas propuestas en la región, Cohen y Franco (2006) justifica la corresponsabilidad en las políticas públicas sosteniendo el análisis de que la pobreza extrema suele transmitirse intergeneracionalmente, desde el momento del nacimiento de una persona, implicando problemas nutricionales para los niños, ingreso tardío al sistema educativo, deserción escolar por la necesidad de inserción en el mercado de trabajo entre otros aspectos. De este modo, para estos autores, las políticas con corresponsabilidad aportan a romper el círculo perverso de transmisión de la pobreza. Se entiende, entonces que la idea de la corresponsabilidad

"establece, en realidad, que el Estado asume el deber de procurar la transferencia y los bienes y servicios adecuados, y la familia beneficiaria se compromete, a su vez, a hacer uso de estos últimos; marca diferencias respecto de otros programas para superar la pobreza que se basaban en la entrega de productos (bienes y servicios) sin exigir nada en contrapartida.

Esto implica que las prestaciones están condicionadas; vale decir, que se entregan solo si el beneficiario cumple los compromisos asumidos con el Programa." (2006: 44) ${ }^{3}$.

Finalmente, en la enumeración de los argumentos acerca de las implicaciones 
de la corresponsabilidad en las políticas sociales, es oportuno mencionar los aportes de Cecchini y Madariaga, miembros de la CEPAL, quienes asocian la presencia de condicionalidades leves, donde es negociado con las familias y no asociado a sanciones y suspensiones en el acceso, a una intervención de carácter promocional, en tanto la corresponsabilidad permitiría que los usuarios del programa sean partícipes del proceso de mejoramiento de sus condiciones de vida. De este modo, afirman los autores que

las condicionalidades están orientadas a la participación de los usuarios en distintos programas públicos, según la dimensión trabajada, y a la atención de los requerimientos específicos de cada programa. En este contexto, la flexibilidad en la definición de las condicionalidades está dada por la jerarquía que asignan las propias familias a sus necesidades y los contratos de cumplimiento de los mínimos, pero no por la definición de cuáles son las dimensiones a trabajar, que vienen predefinidas. La flexibilidad de este tipo de condicionalidades supone, además, la posibilidad de adaptación de la oferta de servicios y programas a las realidades particulares de estas familias (2011: 90).

Ahora bien, estos argumentos que sustentan los Programas de Transferencia de Renta Condicionada en América Latina exigen ser analizados en el marco de las tendencias socio-políticas contemporáneas, donde, como es mencionado anteriormente, aparece interpelada la noción de ciudadanía. En este sentido, es posible afirmar que la corresponsabilidad en los términos arriba mencionados es vinculada a nuevas manifestaciones del pensamiento liberal, el cual expresa tanto posturas intelectuales como formas particulares de intervenir sobre la "cuestión social".

Un referente intelectual que merece ser mencionado en estas discusiones es el pensador contemporáneo francés Pierre Rosanvallon, quien afirma la presencia de una "nueva cuestión social" caracterizada por la crisis de los principios organizadores de la solidaridad y la concepción de derechos sociales. A partir de identificar la crisis de la sociedad salarial vivenciada en Europa, principalmente en Francia, en la década de 1990, el autor menciona que "los antiguos mecanismos productores de solidaridad, en primer lugar están desintegrándose de manera probablemente irreversible. Dichos mecanismos, asentaban en el sistema de los seguros sociales: la solidaridad fundaba en la mutualización creciente de los riesgos sociales, de modo que el Estado providencia se identificaba con una especie de sociedad aseguradora" (2004:10). 
Además, lejos de identificar las causas de los procesos de expulsión del mercado de trabajo de amplios sectores de la población en tendencias societales, afirman la necesidad de avanzar en la explicación de situaciones particulares, donde, por ejemplo, "las variables explicativas de la duración de la desocupación, se sabe hoy, deben ser buscadas en un lugar distinto del de los meros datos sociológicos habituales. Es preciso comprender las cosas en un nivel mucho más fino e individualizado. [...] Lo que debe describirse para comprender qué la desocupación de larga duración son situaciones y trayectorias individuales y no de grupos o poblaciones" (Ibíd.: 191).

Como consecuencia, las causas de las distintas expresiones de la "nueva cuestión social" deben buscarse en variables de comportamiento de las personas, la singularización de los procesos sociales tiene como correlato una postura alternativa al llamado Estado de Bienestar, el cual es caracterizado como obsoleto. El anhelo es, entonces, la instauración de un Estado providencia activo, donde la política pública es asociada a la exigencia de contrapartidas, instalando de este modo una nueva relación de derecho/obligación. Aquí no se trata sólo del merecimiento de la asistencia, sino, también, de la necesidad de incidir sobre las conductas de las personas, en tanto las causas de la "nueva cuestión social" encontrarían en las trayectorias individuales.

La expresión y materialización histórica más acabada de esta postura es encontrada en el denominado workfare, cuyo ejemplo fundamental lo constituye la experiencia estadounidense, aunque muchos de sus elementos aparecen en países europeos y del resto de América, donde surgen políticas de gestión de comportamiento, políticas que implican orientaciones que incluyen desde la presión educativa hasta la intervención sobre la forma de la estructura familiar.

El welfare to work -o su contracción workfare-centrado en la premisa que el bienestar es alcanzado mediante la inserción laboral de las personas, por lo cual la ciudadanía es vinculada a la obligatoriedad de obtener un empleo para poder percibir algún tipo de prestación social. Como correlato, la recepción de una prestación es asociada al cumplimiento de condicionalidades, donde la asistencia social es mercantilizada y la ciudadanía es erosionada y deviene en contrato que exige el cumplimiento de obligaciones por parte de los sujetos (Moreno Márquez, 2008).

Haciendo un examen crítico de las experiencias vinculadas al workfare, Handler (2003) afirma que el discurso conservador que lo sustenta insiste en la 
necesidad de promover cambios de conducta en los desocupados a fin de no erosionar la ética del trabajo. Como menciona este autor, esta propuesta se basa en la estrategia de "trabajar es lo primero", que supone: 1) la existencia de puestos de trabajo para el que quiera trabajar; 2) las personas deben aceptar cualquier trabajo, con la posibilidad, siempre presente, de ascender en la escala laboral; 3) los beneficiarios de la asistencia social no tienen motivación o incentivo para abandonar el beneficio e insertarse en el mercado de trabajo; y 4) siempre es posible el paso de los programas estatales al trabajo.

Tal como afirma Rodríguez Enríquez (2011), la noción de corresponsabilidad, asociada al mérito y a manifestar interés por salir de la situación de pobreza, se vincula con la idea afianzada durante el neoliberalismo que centra la responsabilidad individual de las personas pobres sobre su situación de pobreza y las posibilidades de superarla.

La idea de un contrato mutuo entre la familia, o algún miembro en particular y el Estado erosiona la idea de asistencia social como derecho y desvincula la cotidianidad de las personas de las tendencias económicas, políticas y sociales que se constituyen en explicaciones socio-históricas de los procesos sociales que interpela su reproducción permanentemente.

Este proceso de corresponsabilidad de la política social viene acompañado de un segundo mecanismo fundamental, la familiarización, donde se legitima la transferencia de responsabilidades del Estado a las familias y/o comunidades.

\section{La familiarización como fundamento de la política pública}

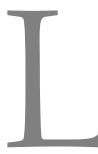

a preocupación por la familia en el proceso de gestión de la fuerza de trabajo encuentra eco en las distintas perspectivas teórico-analíticas, dando cuenta de posturas y visiones disímiles acerca de su vinculación con la sociedad y su funcionalidad socio-histórica (Cicchelli y Cicchelli, 1999). En términos generales, se puede afirmar que la importancia de la familia se fundamenta por ser la encargada de garantizar la fuerza de trabajo para la producción y reproducción del capital. Es necesario promover que la familia garantice, tanto en el plano objetivo como subjetivo, la presencia de trabajadores libres dispuestos a ser parte del proceso de venta de su fuerza de trabajo. El proceso de reproducción de la fuerza de trabajo, siguiendo a Torrado (2003), hace uso de la familia tanto para sufragar los costos de reproducción de la fuerza de trabajo, como así también para asegurar el disciplinamiento de sus miembros ${ }^{4}$.
${ }^{4}$ Agrega Torrado al resesta institución depende la reproducción biológica, la preservación y perpetuacion del orden social, cultural y econó mico, así como la gestión fuerza de trabajo de la chos y potentes mécanismos sociales y políticos se ponen en marcha en cada situación concreta para asegurar dicho con- 
En su estudio de los distintos regímenes de bienestar, por ejemplo Andersen plantea que la esencia del régimen de radica en la articulación de estatus y familiarismo. Este último es considerado como "la combinación de la protección social sesgada a favor del varón cabeza de familia y el carácter central de sadora couidos en última instancia res ponsable del bienestar de sus miembros (principio de subsidiariedad) [... Aparte de la obligación egal, existe una sistemáica falta de disposición cios sanitarios $y$ cuanmas familiarista sea el estado del bienestar, menos generosos serán os subsidios familiares" (2000: 113).

${ }^{6}$ Cursivas nuestras.
Ahora bien, esta preocupación y consideración por las relaciones familiares que subyace a las intervenciones estatales sobre la "cuestión social" en general y a las políticas sociales en particular, no es homogénea y puede ser analizada como resultado de la correlación de fuerzas en un momento histórico y lugar determinado. La consideración de "el otro" en la política social expresa, entonces, el alcance socio-histórico de la ciudadanía, y, con ello, una postura acerca la "responsabilidad del destino" de las personas en una tensión entre individuo y sociedad, donde la familia se constituye en una mediación fundamental.

En este proceso, podemos definir a la familiarización como un mecanismo que configura las políticas asistenciales, determinando un modo particular de intervención sobre la "cuestión social", focalizándose en la familia. A lo largo de la historia, esta estrategia de intervención estatal ha estado presente de diferentes formas, configurando gran parte de las políticas públicas, en determinados contextos políticos, económicos y sociales. En la actualidad, en el marco de tendencias neo-familiaristas se produce la transferencia de responsabilidades hacia las familias, concibiendo a éstas como una unidad económica y política para la resolución de los distintos problemas del modelo (De Martino, 2001) .

De acuerdo a lo anteriormente mencionado, corresponsabilidad y familiarización constituyen mecanismos articulados y expresan en las distintas experiencias de Programas de Transferencia de Renta Condicionadas implementados en los países de América Latina. En el ya mencionado trabajo de Cecchini y Madariaga afirman, por ejemplo, que los objetivos de estos programas están orientados a

... transformar y detener la transmisión intergeneracional de la pobreza mediante el desarrollo de las capacidades humanas en las familias ${ }^{6}$ más vulnerables. Es por esto que los PTC, entregan transferencias monetarias directas y establecen condicionalidades como la asistencia a la escuela y a los controles de salud. De esta forma, los programas no sólo contribuirían a la reducción contingente de la pobreza de ingresos, sino también a la formación de capacidades humanas, un activo clave del desarrollo sostenible y del progreso en nuestras sociedades. (2011: 5 - 6)

Como vemos, el acento de estos programas está puesto en la familia, como la única institución responsable de su situación, pero además no se habla de 
cualquier familia, sino que se focaliza en "familias vulnerables". Siguiendo este planteamiento, a través de la entrega directa de dinero se le exige a la familia, bajo el discurso de la corresponsabilidad, una "contraprestación" o "condicionalidad" que generalmente se asocia al cumplimiento con la asistencia a la escuela y los controles de salud, para propiciar el desarrollo de las "capacidades humanas".

Siguiendo con el análisis del documento de la CEPAL, se considera que la estructura básica de los programas es la entrega de recursos monetarios y no monetarios a familias en situación pobreza o pobreza extrema que tienen uno o más hijos menores de edad, con la condición de que estas cumplan con ciertas conductas asociadas al mejoramiento de sus capacidades humanas (Ibíd., 13). Una vez más, vuelven a enfatizar en las obligaciones y las conductas que deben cumplir las familias en función del beneficio que perciben por parte del programa, incluyendo en esta estructura a los hijos menores, que serían el "soporte" educativo que se intenta formar para las futuras generaciones.

Articulando el proceso de familiarización con la comunitarización (Danani, 2005), como par que conjuntamente implican la disminución de la presencia del Estado responsable de la asistencia social, la CEPAL en el año 2006 explicita que

... la importancia de la incorporación del fomento del capital social como uno de los aspectos de los programas para la superación de la pobreza que puede propiciar una mayor inclusión social, porque supone prestar especial atención a las redes que permiten vincular a los sectores pobres entre sí y con los demás sectores de la población. Concretamente, se apunta a modificar el alcance de las redes sociales y el grado de asociación entre grupos con distinto tipo de capital social. También significa hacer hincapié en el papel de las relaciones sociales de confianza, reciprocidad y cooperación, en la sostenibilidad de iniciativas comunitarias y en las estrategias desarrolladas para mitigar los efectos de la pobreza. Por otra parte, el desarrollo del capital social en los programas también apunta a que los agentes tengan una mayor participación y un mayor protagonismo en la solución de sus problemas (2006:176).

La lógica comunitarista en los Programas de Transferencia de Renta Condicionada se desarrolla a través del discurso de la incorporación del capital social de las familias para la "superación" de su condición de pobreza. Este discurso, considera que al fortalecer las redes sociales de ayuda mutua, 
cooperación y confianza entre los mismos sectores y con otros, se alcanzaría una mayor inclusión social. Además, el desarrollo de este tipo de redes haría que la familia sea partícipe de la solución de sus problemas, desde la lógica de fomentar su protagonismo. Este tipo de argumentos, muestran un modo particular de intervención sobre la "cuestión social", donde paulatinamente la supuesta corresponsabilidad se desvanece en un horizonte que supone un Estado desvinculado de las causas de las situaciones que interpelan la vida cotidiana de las personas y, por lo tanto, promueve la desvinculación e invisibilización del Estado de las responsabilidades y obligaciones que debe cumplir con todos los ciudadanos, para que sea la familia quien deba hacerse responsable de su situación.

Profundizando la lectura analítica, al poner en relación los artículos del año 2006 y 2011 de la CEPAL, vemos algunas continuidades en los planteos que sustentan los programas considerados. Ejemplo de ello es que se sigue sosteniendo que la unidad de intervención de los Programas de Transferencia de Renta Condicionada sea la familia en su conjunto, asignándole un rol protagónico a la mujer a través de la recepción de las transferencias monetarias, asociando a que las madres serán las encargadas del bienestar familiar y de sus hijos en particular (2011: 13, 14 y 2006: 166).

Estas tendencias familiaristas se expresan también en el documento del Banco Mundial donde se considera que los objetivos particulares de la madre pueden estar vinculados estrechamente con los de todos sus hijos o quizás especialmente con los de sus hijas. Esta vinculación con frecuencia está dada como una justificación para efectuar la transferencia monetaria a la madre y no al padre, como es la práctica común en la mayoría de los programas de Transferencias Monetarias Condicionadas (TMC), aspecto que se profundizará en el próximo apartado.

Siguiendo con la misma lógica argumental, el Banco Mundial plantea que las transferencias monetarias condicionadas

son programas que transfieren dinero, generalmente a familias pobres, con la condición de que estas efectúen inversiones especificadas de antemano en el capital humano de los hijos. Las condiciones de salud y nutrición requieren generalmente chequeos periódicos, control del crecimiento y vacunas para los niños menores de cinco años de edad; la atención perinatal para las madres y su asistencia a charlas periódicas de información sobre la 
salud. Las condiciones de educación usualmente incluyen la inscripción escolar, la asistencia al colegio entre el 80 y el 85\% de los días escolares y ocasionalmente alguna medida de desempeño. La mayoría de los programas de TMC transfieren el dinero a la madre de la familia o, en algunas circunstancias, al estudiante (Fiszbein y Schady; 2009: 1).

Una vez más, nos encontramos que la población objetivo de estos programas son familias que se clasifican como "pobres" que deben "invertir" en el desarrollo humano de sus hijos, los cuales son considerados en los términos de capital humano. Además, el hecho que las condiciones se centren en construir el capital humano de los hijos (y no simplemente apoyar a los padres) añade a la aceptabilidad política de las transferencias monetarias condicionadas como instrumento para promover "oportunidades", ya que es difícil culpar a los niños por ser pobres (Ibíd.: 10). Este discurso, que hace hincapié en la formación educativa y cuidados de la salud de los niños y madres (durante la etapa perinatal), lleva implícita la necesidad de reproducir un modelo familiar determinado por la tendencias familiaristas que definíamos anteriormente, y formar además futuros vendedores de la fuerza de trabajo, para mantener y reproducir las relaciones sociales capitalistas.

Dichas condiciones de los programas se justifican, desde el Banco Mundial, con el argumento que hay familias que no invierten lo suficiente en el capital humano de sus hijos. Por ejemplo, si mantienen creencias incorrectas sobre los rendimientos de estas inversiones, si existe un "altruismo incompleto" entre los padres y los hijos, o si existen grandes externalidades a las inversiones en salud y educación (Ibíd.: 2). De esta forma, se justifica la necesidad de introducir condicionalidades (como los controles de salud y la asistencia a la escuela) para las familias receptoras de los programas, desde la lógica que estas no cumplen con ciertas pautas de comportamiento funcionales al sistema y son "disruptoras" del orden social, de ahí la necesidad que cumplan con ciertos requisitos que las llevarán a incorporar hábitos y conductas "normales" para la vida en sociedad. Incluso se habla de plantear la idea de penalización por no cumplir la condicionalidad, penar mediante el no acceso a una prestación que implica acceder a un derecho (Ibíd.: 31). Este análisis, asocia contradictoriamente la penalización (por el no cumplimiento de la condicionalidad) con las prestaciones como derecho, trastocando la noción de ciudadanía, re-vulnerabilizando un derecho históricamente conquistado.

Continuando con la lectura analítica nos encontramos con un postulado del 
Banco Mundial donde se manifiesta que

...la esencia de un programa es que las familias se aseguren de que los hijos utilicen los servicios de salud y educación y, si estos no están disponibles, se excluye a las familias del programa. Algunos programas, al menos en los primeros años, intentaron cubrir zonas pobres indicadas por un mapa de pobreza, pero efectuaron operaciones solo en las zonas en que los servicios fueron estimados como accesibles e intencionalmente excluyeron a todos los pobres que vivían en zonas sin una capacidad mínima de servicios. (Ibíd.: 83)

En este postulado se visualizan fundamentos netamente excluyentes, planteando que si no hay espacios en los servicios de salud y educación para garantizar las condicionalidades, se deja afuera a las familias. Esto da cuenta, entonces, que se prioriza más cumplir con los requisitos del programa (que tienden a reducir cada vez más la población objetivo), que generar estrategias que busquen incorporar a todas las familias.

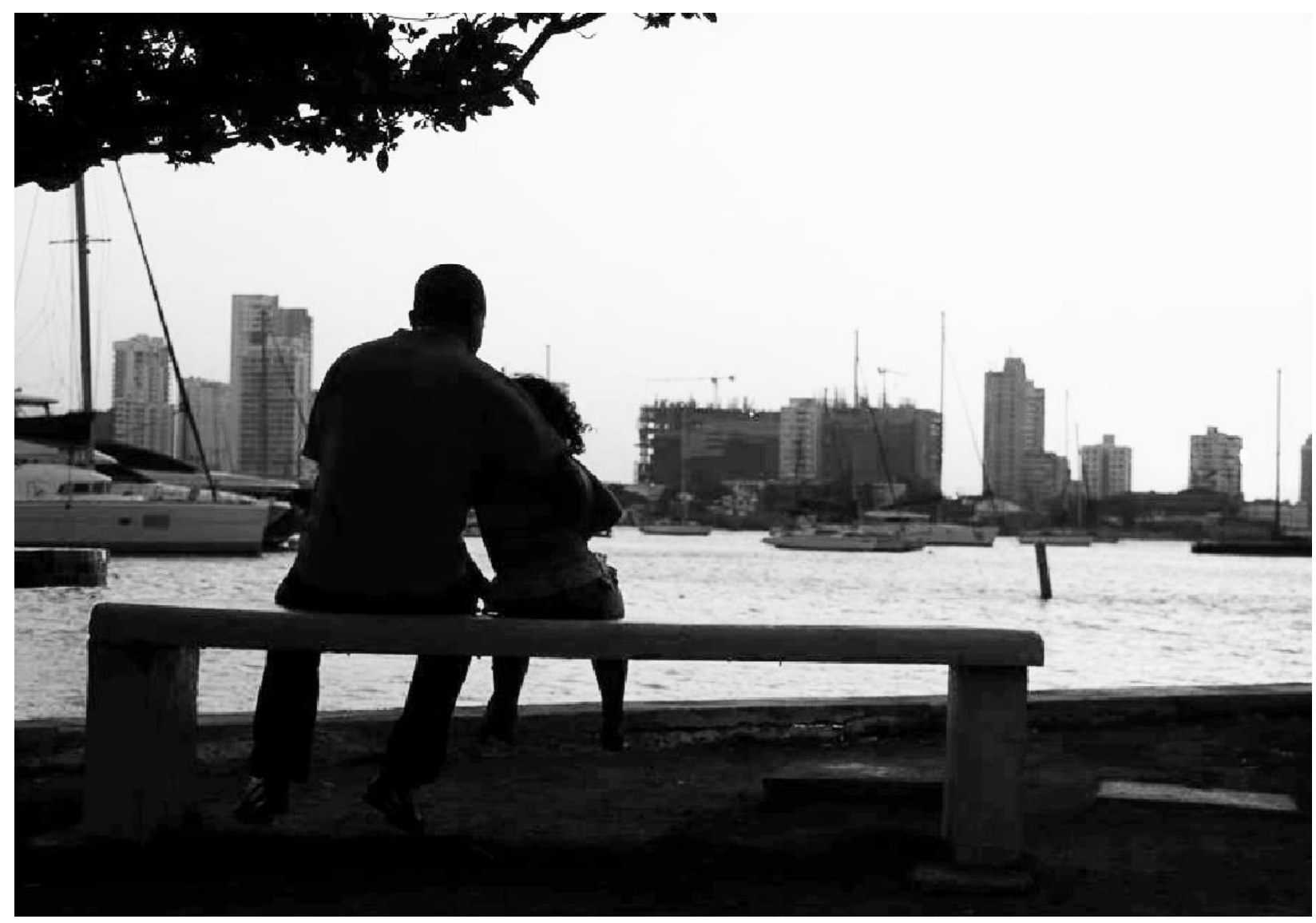


Como se hizo mención anteriormente, los mecanismos de corresponsabilidad y familiarización vienen asociados a una tercera estrategia de la política social, la maternalización, en donde se plantea como prioritaria la intervención con la mujer, quien se constituye en objeto y sujeto de las intervenciones realizadas por los sectores dominantes (Grassi, 1989). Sobre este mecanismo trabajaremos los principales elementos en el siguiente apartado.

\section{La maternalización de los procesos sociales: principales elementos}

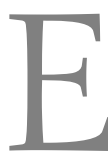
n el marco de la configuración de los procesos de intervención sobre las distintas expresiones de la "cuestión social" en los Estados capitalistas, el proceso de transferencia de responsabilidades a los ciudadanos, mediante los discursos de la corresponsabilidad y las estrategias de familiarización, se han conformando articulando la relación del Estado, en sus distintas expresiones, con un miembro particular de la familia sobre la cual se interviene: la mujer.

Recuperando discursos y prácticas socio-históricas que suponen diferencias entre el mundo del trabajo y el mundo de la casa y la familia, con sus consecuentes repercusiones en el cotidiano de hombres y mujeres, pues la participación legítima se define de manera diferencial para unos y otros, respectivamente. Al respecto, Cicchelli y Cicchelli (1999) plantean que asociado a una visión de las relaciones sociales burguesas caracterizadas por la conjunción de elementos asociados a los modelos educativos definidos en función del sexo, la asignación de la mujer al hogar y la atribución al hombre del papel de proveedor, se plantea en el siglo XIX la tarea de educar a los sexos, donde un aspecto fundamental es "moralizar a la obrera transformándola en ama de casa: esa es la primera orientación del proyecto de socialización de las clases trabajadoras y, más particularmente, de las relaciones conyugales" (Cicchelli y Cicchelli, 1999: 30).

Esta tendencia es reforzada por las intervenciones estatales y por prácticas profesionales diversas, como la medicina, la economía doméstica, entre otras, donde, como afirma Nari, "la diferencia sexual que homogeneizaba a las mujeres tomando como eje su capacidad biológica y cultural de gestar, criar, cuidar y educar a otros seres humanos. La maternidad, hecho y proceso biológico-cultural que involucraba todas estas características, también era esgrimida como la base que justificaba y legitimaba la subordinación social de las mujeres, sea en términos de inferioridad o de necesidad de protección" (2004: 231). 
En este contexto, afirma la autora que la diferencia sexual y la maternalización eran supuestos no problematizados, arraigados y naturalizados en la sociedad. En este punto es importante destacar que si bien la maternidad refuerza la idea de una diferencia sexual basada en la capacidad física de procrear, pero fundamentalmente "espiritual" de las mujeres para ser madres, entendiendo la aceptación como "función natural" femenina, esta condición biológica no justifica la desigualdad jurídica, social y política ni el reclamo público y político de igualdad de derechos al hombre.

De este modo, este clima maternalista imperante, durante las primeras décadas del siglo XX, pretende construir una "nueva mujer" entre la maternidad y el trabajo, la educación y la política. La intervención de los sectores dominantes poco a poco fue imposibilitando el intento de instalar la participación de la mujer en la educación, el trabajo y en el espacio público, donde esta no aparece considerada como ciudadana sino como mujer-madre.

Teniendo en cuenta la experiencia de los ya mencionados Programas de Transferencia de Renta Condicionada implementados en América Latina y analizados por organismos internacionales explicitados anteriormente, se observa que la unidad de intervención se centra en la familia en su conjunto, y dentro de la misma se le otorga a la mujer un papel fundamental, siendo estas las encargadas por un lado de "distribuir lo mejor posible" los recursos monetarios transferidos por el programa para mejorar el bienestar de su familia en general y de los hijos en particular, y, por otro lado, son las responsables del cumplimiento de las condicionalidades obligatorias como destinatario partícipe del programa (Cecchini y Madariaga, 2011). Al respecto la CEPAL plantea:

"El desafío que se debe enfrentar en estos programas es la mayor incidencia de la pobreza en los hogares encabezados por una mujer o que se encuentran en la fase de expansión del ciclo de vida familiar. En particular, las mujeres que encabezan hogares con varios hijos pequeños suelen enfrentar conflictos entre sus obligaciones de cuidado de los niños, las tareas del hogar y el imperativo de trabajar para mantener la familia. Estos conflictos deberían tenerse en cuenta en la formulación de los programas, a fin de que estos beneficien a quienes más los necesitan" (2006:163).

La decisión política de los Programas de Transferencia de Renta Condicionada de otorgar a la mujer una centralidad absoluta en relación a constituirse 
como la única responsable de ejecutar las obligaciones de contraprestación del programa, promueve un proceso de "maternalización" de las políticas sociales como sujetos titulares del cumplimiento del mejoramiento de las denominadas capacidades humanas y sociales de los ancianos, discapacitado y en especial de los hijos que están a su cargo.

Por su parte, en los fundamentos del informe del Banco Mundial sobre investigaciones relativas a las Políticas de Desarrollo del año 2009, en lo que respecta a la relación entre el Estado y la familia, se aprecia como la mujer beneficiaria del programa es la encargada de optimizar los recursos y desarrollar el capital humano de sus hijos, como así también el argumento "del paternalismo-buen comportamiento", corre del eje la responsabilidad y función que les cabe a ambos progenitores, dentro de la estructura familiar y de los Programas de Transferencia de Renta Condicionada, " al maternalismobuen comportamiento". Se afirma que si bien la noción de paternalismo es muy antigua y en la actualidad es considerada por los economistas como obsoleta para promover las formas condicionales de redistribución, esta se podría justificar en la actualidad ya que, según Currie y Gahvari plantean que "el paternalismo se relaciona íntimamente con la idea de bienes y deseos meritorios, y puede ser una de las razones clave para la intervención del gobierno" (2007: 6 en: Fiszbein y Schady; 2009: 53).

De acuerdo a lo expresado se puede inferir que, junto a los procesos de corresponsabilidad y familiarización de las estrategias por parte del Estado para dar respuesta a las manifestaciones de la "cuestión social", se torna necesario "maternalizar" la implementación de las políticas sociales a través de los Programas de Transferencia de Renta Condicionada. Las autoras Martínez y Voorend (2008) expresan que estos programas reproducen una visión "maternalista" del acceso a los recursos públicos y que a diferencia de programas asistenciales previos, el diseño explicita una contraprestación que exige y demanda tiempo de trabajo femenino.

La fundamentación de estos planteamientos se basa en argumentos relacionados a valoraciones asociadas al rol y funciones que realizan las mujeres en el ámbito del hogar, en relación al cuidado de los hijos/as. Entendiendo que es el propio Estado el que establece este enfoque maternalista, estrategia que, como hemos visto, no es nueva ni novedosa en el diseño de programas sociales, ya que la "feminización de las estrategias para hacer frente a la escasez de 
recursos" es una práctica conocida históricamente en la implementación de las políticas sociales establecidas de America Latina.

Ahora bien, los dos elementos que constituyen las principales condicionalidades de los Programas de Transferencia de Renta Condicionada refieren a la educación y salud, ambos considerados bienes meritorios, por lo cual el Estado interviene para maximizar los recursos y alcanzar un mayor impacto en la población pobre beneficiaria. Por ello, cuando el Estado opta por una población objetivo específica y no por otra, trae aparejado entender que los sectores seleccionados se presentan como una alternativa que le permitirá concentrar los recursos financieros en unidades de inversión "mas barata" consiguiendo un impacto mayor per-cápita en relación al desarrollo del capital humano y social.

Es interesante apreciar, por su parte, como en los mismos planteos de los documentos analizados, el papel central que las mujeres tienen en los programas, no solo como administradoras sino como responsables del cumplimiento de las contraprestaciones, presentan contradicciones, en tanto que por un lado se afirma sobre el denominado empoderamiento que estas acciones tendrían sobre las mujeres, al tener mayor capacidad de participación en la comunidad, mientras que, por el otro, se afirma que esta estrategia se diluye en la medida de que en la totalidad de los Programas de Transferencia de Renta Condicionada la participación de la mujer está orientada al desarrollo del llamado capital humano de sus hijos y no de ellas mismas (Cecchini y Madariaga, 2011).

Al respecto la CEPAL (2006) marca las tendencias que existen al respecto: Por una parte, se sostiene que la percepción de ingresos monetarios les permitiría a las mujeres modificar su papel pasivo o subordinado dentro de la familia, mientras, que, por la otra, se critica la implementación des exigencias que se plantean a las mujeres en tanto su participación aparece como promotoras de los programas. Una postura problematizadora de las tendencias en estas intervenciones intenta dar cuenta que estos programas terminan recargando y reforzando las actividades de cuidado que tradicionalmente han limitado la inserción de la mujer en el mercado del trabajo (Pautassi y Zibecchi, 2010), en detrimento de la distribución igualitaria de tales actividades al interior de las relaciones familiares. Se refuerza la división tradicional entre mundo del trabajo y mundo de la casa, donde institucionalmente se legitima el discurso que circunscribe a la mujer al interior del segundo. 
Por otro lado, considerando que, como hemos visto, las llamadas condiciones de elegibilidad en los Programas de Transferencia de Renta Condicionada en su mayoría se asocian a la presencia de niños y niñas en las familias, una vez que estos no cumplen con los criterios etáreos definidos, la familia queda excluida del programa. Como consecuencia, la mujer debe procurar el ingreso económico para su cotidiano en el mercado de trabajo, del cual la misma política pública mantuvo alejada.

\section{Síntesis: sobre las nuevas formas de individualización en la política social contemporánea}

l presente texto procura exponer las particularidades que adquieren los
mecanismos de corresponsabilidad, familiarización y maternalización
en la política social contemporánea, haciendo especial referencia a los Programas de Transferencia de Renta Condicionada, los cuales han constituido una de las intervenciones estatales sobre la "cuestión social" de mayor relevancia en la región de América Latina.

El análisis de documentos emanados por organismos internacionales, como la CEPAL y el Banco Mundial, permite reconstruir las principales tendencias de estos mecanismos, logrando identificar la complementariedad necesaria de cada uno de ellos para con el resto. Es decir, mediante los mecanismos de corresponsabilidad, familiarización y maternalización de la política social reforzando el análisis de los problemas sociales como expresiones de dificultades en el ethos individual (Netto, 2002). Como consecuencia de la misma se produce una negación de la procesualidad socio-histórico y una desvinculación de esas situaciones de aspectos los procesos generales, apelando a la exaltación de dificultades individuales y familiares lo cual demandaría una intervención activa del Estado sobre las capacidades humanas de las personas.

La mercantilización de los derechos sociales, mediante su condicionalidad trastoca el concepto de ciudadanía para amplios sectores de la población, donde el acceso a prestaciones fundamentales para garantizar su reproducción cotidiana se encuentra supeditado al cumplimiento de determinadas exigencias. En esta línea, se considera que la problematización realiza se constituye en un insumo para posteriores reflexiones, propias y ajenas, que abonen al intercambio sobre las implicancias contemporáneas de los Programas de Transferencia de renta Condicionada. 


\section{BIBLIOGRAFÍA}

Arriagada, I. 2007. "Transformaciones familiares y políticas de bienestar en América Latina". En: Arriagada, I. (Coord.). Familias y políticas públicas en América Latina. Una historia de desencuentros. CEPAL-UNFRA, Chile.

Cecchini, S. y Madariaga, A. 2011. Programas de transferencias condicionadas. Balance de la experiencia reciente en América Latina y el Caribe. Naciones Unidas, Santiago de Chile.

CEPAL, 2006. La protección social de cara al futuro: acceso, financiamiento y solidaridad. CEPAL, Chile.

Cicchelli-Pugeault, C. y Cicchelli, V. 1999. Las teorías sociológicas de la familia. Ediciones Nueva Visión, Buenos Aires.

Cohen, E. y Franco, R. 2006. Transferencias con corresponsabilidad. Una mirada latinoamericana. Flacso, México.

Coutinho, C. N. 1997. Notas sobre cidadania e modernidade. Em: Praia Vermelha № 1 - Escola de Serviço Social - Universidade Federal do Rio de Janeiro, Brasil.

Danani, C. 2005. Las políticas sociales de los '90: los resultados de la combinación de individualización y comunitarización de la protección. En: Coloquio Internacional: "Trabajo, conflictos sociales e integración monetaria: América Latina en una perspectiva comparada", Instituto de Ciencias (UNGS)/Institut de Reserche por le Développement/ANPCyT-FONCyT - Buenos Aires.

Danani, C. 2009. "La gestión de la política social: un intento de aportar a su problematización". En: Chiara, M. y Di Virgilio, M. M. Gestión de la política social. Conceptos y herramientas. Prometeo, Buenos Aires.

De Martino, M. 2001. Políticas sociales y familia. Estado de bienestar y neo-liberalismo familiarista. En Revista Fronteras. Departamento de Trabajo Social. FCS - UDELAR. Nro. 4, Montevideo.

Esping-Andersen, G. 2000. Fundamentos sociales de las economías postindustriales. Ariel, Barcelona.

Fiszbein, A. y Schady, N. 2009. Transferencias monetarias condicionadas. Reducción de la pobreza actual y futura. Banco Mundial, Washington.

Grassi, E. 1989. La mujer y la profesión de asistente social. El control de la vida cotidiana. Editorial HVMANITAS, Buenos Aires.

Handler, J. F. 2003. "Ciudadanía social y workfare en Estados Unidos y Europa Occidental: de status a contrato". En: Lindenboim, J. y Danani, C. Entre el trabajo y la política. Las reformas de las políticas sociales argentinas en perspectiva comparada. Biblos, Buenos Aires.

Iamamoto, M. 1997. Servicio social y división del trabajo. Cortez Editora, San Pablo.

Mallardi, M. 2012. Cuestión social y cotidiano. Implicancias objetivas y subjetivas de la sociabilidad capitalista. CEIPIL, Argentina. 
Martínez, J. y Voorend, K. 2008. Transferencias condicionadas e igualdad de género: ¿Blancos, negros o grises?. En: Revista de Ciencias Sociales. San José, Costa Rica. Universidad de Costa Rica.

Moreno Márquez, G. 2008. La reformulación del Estado del bienestar: el workfare, las políticas activas de empleo y las rentas mínimas. Revista EKAINA, Madrid.

Nari, M. 2004. Políticas de maternidad y maternalismo político. Biblos, Buenos Aires.

Netto, J. P. 2002. Capitalismo monopolista y servicio social. Cortez Editora, San Pablo.

Oliva, A. 2007. Trabajo social y lucha de clases. Análisis histórico de las modalidades de intervención en Argentina. Imago Mundi, Buenos Aires.

Pautassi, L y Zibecchi, C. 2010. La provisión de cuidado y la superación de la pobreza infantil. Programas de transferencias condicionadas en Argentina y el papel de las organizaciones sociales y comunitarias. CEPAL, Chile.

Rodríguez Enríquez, C. 2011. Programas de transferencias condicionadas de ingreso e igualdad de género ¿Por dónde anda América Latina? CEPAL, Chile.

Rosanvallon, P. 2004. La nueva cuestión social. Repensar el Estado Providencia. Manantial, Buenos Aires.

Soldano, D. y Andreanacci, L. 2005. "Aproximación a las teorías de la política social a partir del caso argentino". En: Andrenacci, L. (Comp). Problemas de política social en la Argentina contemporánea. Prometeo, Buenos Aires.

Torrado, S. 2003. Historia de la familia en la Argentina moderna 1870-2000. Ed. De la flor, Bs. As. 